\title{
FLARE ACTIVITY OF THE SUN AND VARIATIONS IN ITS UV EMISSION DURING CYCLE 24
}

\author{
E.A. Bruevich, G.V. Yakunina \\ Lomonosov Moscow State University, Sternberg Astronomical Institute, \\ Universitetsky pr., 13, Moscow 119992, Russia \\ e-mail: red-field@yandex.ru,yakunina@sai.msu.ru
}

\begin{abstract}
The flare activity and the ultraviolet emission of the sun during its 24-th cycle are analysed. As compared to cycles 21-23, where the most powerful flares were observed during the decay phase, in cycle 24 the greatest number of powerful flares $(>X 2.7)$ occurred in the rising phase and at the maximum with the exception of the two largest flares of cycle 24 X9.3 and X8.2 in September 2017. We showed that regression fits of solar UV indices to the overall radiation level from the sun are substantially different for cycle 24 compared to cycles 21-23. It is found that for the flare of August 9, 2011 (SDO and GOES-15 observations), the flare propagates in a direction from the upper corona to the transition region and to the chromosphere. A study of the N-S asymmetry in the distribution of the flares in cycle 24 reveals a strong predominance of flares in the N-hemisphere in 2011 and in the S-hemisphere in 2014. It is also found that during cycles 23 and 24, the delays in the onset of proton events relative to the onset of the flares that cause them have a distribution with a distinct maximum corresponding to a delay of 2 hours for protons with energies $>10 \mathrm{MeV}$, as well as for those with energies $>100 \mathrm{MeV}$.
\end{abstract}

Key words. Sun: cycle 24: flares: flare index: variations in UV emission: proton events.

\section{Introduction}

Analyses of the flare activity and of variations in the UV emission of the sun are important for research on solar and terrestrial physics. The accelerated particles generated by powerful flares have a fundamental influence on space 
weather and have many practical applications relating to the study of physical processes in the heliosphere and in the earth's atmosphere.

\section{Features of activity cycle 24}

The current solar activity cycle (No. 24) is the weakest solar cycle over the last 100 years or more. At present the transition to the following activity minimum between cycles 24 and 25, which is expected in roughly 2018-2019, is taking place.

Solar cycle 24 continues the trend of recent years of a reduction in the number of sunspots since cycle 21, which reached its maximum in roughly 1980 (see Fig. 1). Many scientists at NASA who are involved in the prediction of solar activity assume that cycle 25 will be roughly the same or weaker than 24. The variations in the solar activity have been modelled numerically [1] using observational data on the number of sunspots in the period 1750-2050.

The dynamo theory was used to solve the evolution equation for a number of relative sunspot number (SSN) with the mechanism for magnetic field formation in sunspots taken into account. The model results [1] correlate well with observations and predict a prolonged period of low activity up to 2050 similar to the Dalton minimum.

Fig. 1 shows the variations in the number of sunspots over 300 years. The current cycle 24 is clearly one of the lowest and is comparable to the activity during the epoch of the Dalton minimum. It is expected that cycles 25 and 26 will be similar to cycle 24 .

\subsection{Solar activity in the ultraviolet range and flare ac- tivity during cycle 24}

Studies in the ultraviolet (UV) are an important part of research on the development of solar flares. The UV part of the electromagnetic spectrum covers the range from 5 to $400 \mathrm{~nm}$. UV photons are absorbed in the upper layers of the atmosphere; short-wavelength fluxes of solar radiation cause

ionization and dissociation of atmospheric species and lead to formation of the ionosphere. The solar UV emission is formed in the upper chromosphere 


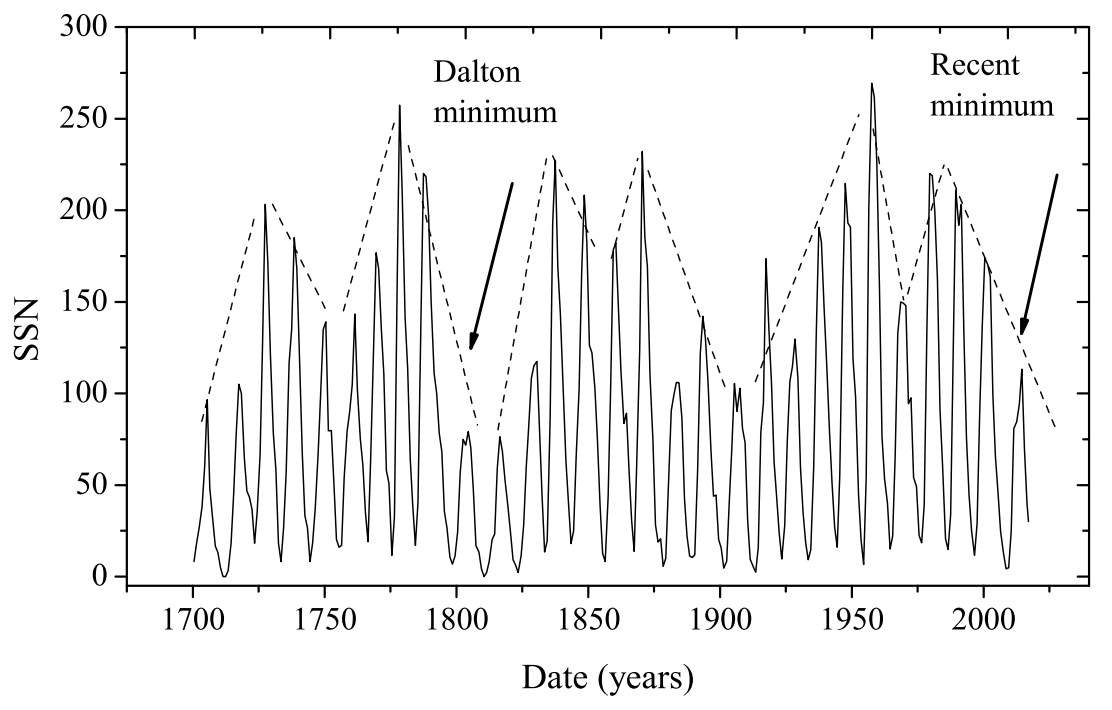

Figure 1: Yearly average number of sunspots (SSN) from 1700 to the present.

and the transition region, while X-rays are formed in the corona; the fluxes in these ranges make up a comparatively small fraction of the overall radiative flux.

As opposed to most stars of the sun's type, the sun is characterized by comparatively low spottedness, moderate flare activity, and a low level of corona emission flux [2,3].

Against this background, even very small variations in the fluxes associated with the appearance and disappearance of groups of spots in the active regions or variations in activity during the solar cycle and large flares can cause significant changes in the UV and X-ray activity indices.

The UV fluxes analysed in this paper vary by tens of percent on time scales ranging from a few minutes to several hours (during solar flares); by a few percent over times ranging from a few days to several months (owing to the movement of active regions over the disk because of the sun's rotation); and by as much as a factor of two over times ranging from a few years to decades (variations in the solar cycle). This variability produces variations 

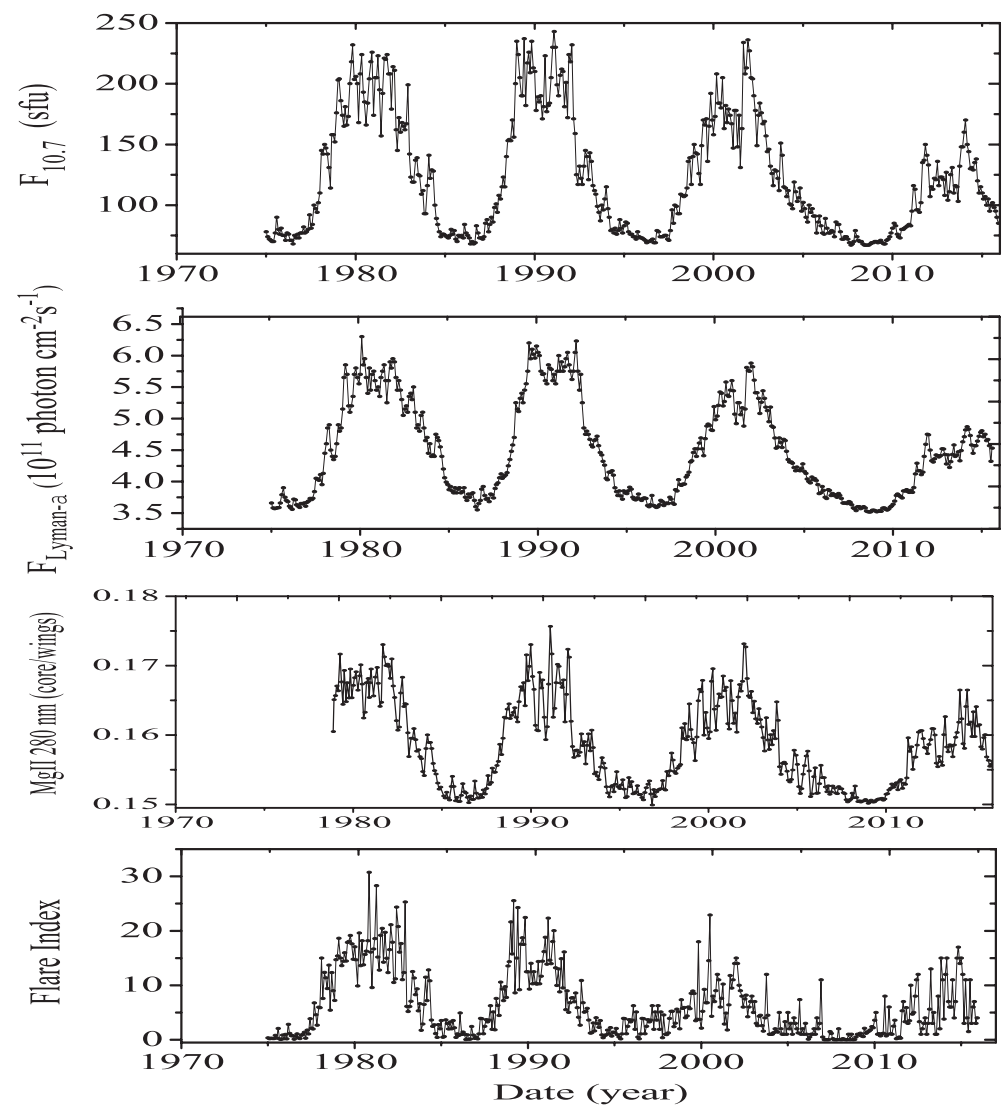

Figure 2: Cyclical variations in the fluxes $F_{10.7}, F_{L y-\alpha}$, and MgII $280 \mathrm{~nm}$ (core/wings) and the flare index (FI) observed during cycles 21-24.

in the earth's ionosphere and upper atmosphere that are similar in amplitude and time. Since the solar UV emission is mainly absorbed in the upper layers of the atmosphere, it is measured using instruments mounted on rockets and satellites.

Because of the related difficulties, for many years different predictions of the UV fluxes were used that employed correlations with the numbers of sunspots and $10.7 \mathrm{~cm}$ radio wave emission $\left(F_{10.7}\right)$. The $F_{10.7}$ flux is now often used as an objective index for the current level of solar activity; this flux is measured in sfu (solar flux units), with 1 sfu corresponding to a flux of $10^{-22}$ $W / m^{2} / H z$. Thus, a comprehensive analysis of observational data on solar UV emission is needed, since the emission in this region of the sun's spectrum 
is the most important input parameter for modelling the state of the earth's ionosphere and determines the space weather in the earth's vicinity.

It has been shown [4] that the relationship between the UV solar activity index and the general activity of the sun based on the value of $F_{10.7}$ differed for cycles 21-23, with changes in the magnitudes of the regression coefficients. The correlation coefficient for a linear regression varies within a single cycle, with two maxima during the rising and decreasing phases and two minima during the cycle minimum and maximum [4].

Fig. 2 shows time series of the ultraviolet activity indices and the flare index compared with a series of $F_{10.7}$ as indicators of the overall activity of the sun's atmosphere. Fig. 2 shows that the current cycle 24 has amplitudes of the UV indices and the flare index that are roughly half those for cycles 21-23. This corresponds to the lower level of solar activity as a whole at the present time.

The goals of this paper are:

(1) to analyse the variations in the UV Lyman alpha $121.7 \mathrm{~nm}$ and MgII $280 \mathrm{~nm}$ lines, as well as of the flare activity of the sun in cycle 24;

(2) to compare the fluxes at wavelengths ranging from soft X-ray (data from GOES satellites for 0.1-0.8 nm) to ultraviolet (data on fluxes in five UV lines from the Solar Dynamics Observatory - SDO) in order to track the propagation of the largest flare in cycle 24 in the solar atmosphere;

(3) to estimate the delay in the onset of proton events relative to the onset of flares in the $0.1-0.8 \mathrm{~nm}$ range associated with these events based on data from the GOES 13-15 satellites.

\section{$3 \quad$ UV fluxes and flare activity in cycle 24}

\subsection{UV radiation flux in the $\mathrm{MgII} 280 \mathrm{~nm}$ line}

The index characterizing the ratio of the fluxes in the center and wings of the atmospheric MgII (280 nm) line has been observed continuously using instruments on satellites since 1978. The core of the doublet MgII 279.56 and $280.27 \mathrm{~nm}$ lines is formed in the chromosphere, but the wings are mainly of photospheric origin. This index is a good indicator of the state of the solar atmosphere. 
A close coupling is observed $[5,6]$ between it and the flux in different lines and ranges of UV emission from the sun, which are important for modelling the state of the earth's atmosphere. The index MgII (core/wings) is measured in relative units. The observations from Ref. 7 are used.

Fig. 3 shows the MgII $280 \mathrm{~nm}(\mathrm{c} / \mathrm{w})$ UV radiation flux as a function of the radio emission flux at a wavelength of $10.7 \mathrm{~cm}$, an indicator of the level of solar activity. The solid points show the dependence for cycles 21-23 and the hollow circles, the dependence for cycle 24. Separate quadratic regression fits are shown for cycles 21-23 and for cycle 24. These fits differ significantly: in cycle 24 the maximum fluxes were roughly half the magnitude of the maxima in cycles 21-23, the spread in the values is larger in cycle 24, and the regression coefficients differ substantially.

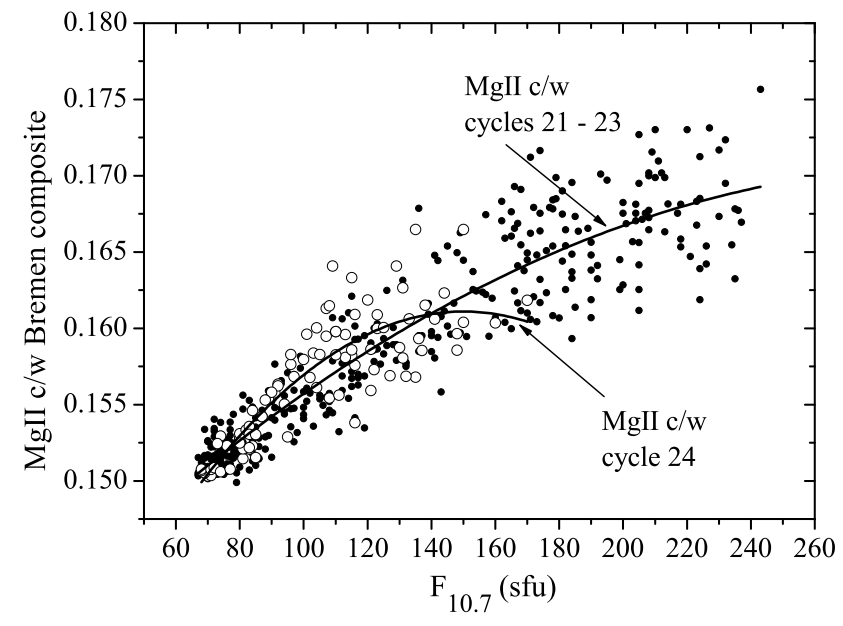

Figure 3: $\mathrm{MgII} \mathrm{c/w}$ as a function of the radio emission flux at $10.7 \mathrm{~cm}$

\subsection{The UV flux in the Lyman $\alpha 121.6 \mathrm{~nm}$ line}

The flux in the Lyman-alpha hydrogen line $\left(F_{L y-\alpha}\right)$ from the entire solar disk is an important indicator that characterizes the state of the chromosphere and the lower part of the transition region [8]. The index $F_{L y-\alpha}$ is measured in units of $10^{11}$ photons $/ \mathrm{cm}^{2} / \mathrm{s}$. The observations from Ref. 9 are used. 
Fig. 4 shows the dependence of the ultraviolet emission $F_{L y-\alpha}$ at 121.6 $\mathrm{nm}$ on the $10.7 \mathrm{~cm}$ radio emission. The solid points show this dependence for cycles 21-23 and the hollow triangles, the dependence of cycle 24 .

Quadratic regression fits are shown here: the regression curves and fits for these two dependences differ more distinctly than for the case of the MgII $280 \mathrm{~nm} \mathrm{c/w}$ index.

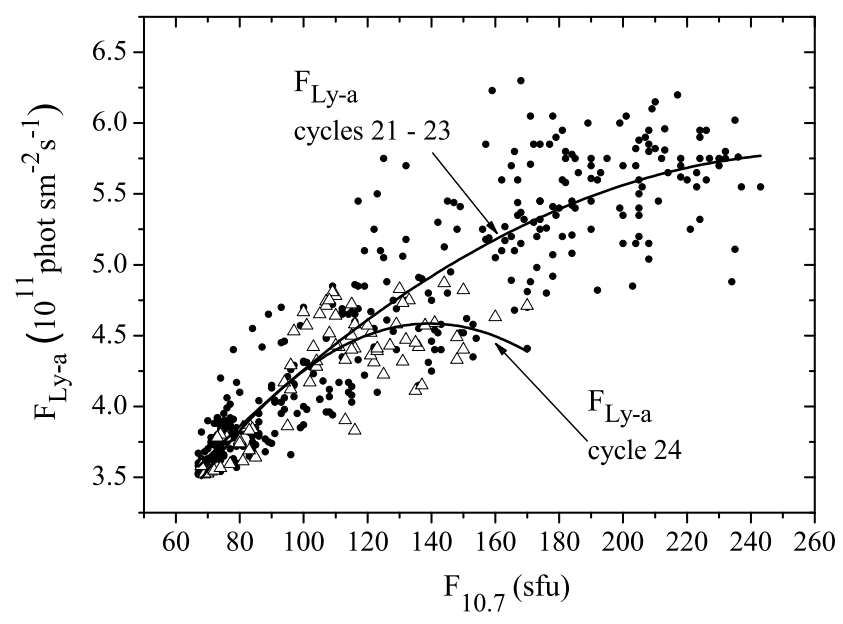

Figure 4: The $F_{L y-\alpha} 121.6 \mathrm{~nm}$ UV radiation flux as a function of the radio emission flux at $10.7 \mathrm{~cm}$

\subsection{The flare index in cycle 24}

The distinctive feature of the time variations in the flare index at the end of the 20th and beginning of the 21st centuries is that by cycle 23 the maximum of FI had fallen by a factor of two compared to cycles 21 and 22. Archived data on FI from 1975 to 2008 are available at

http ://www.ngdc.noaa.gov/stp/space-weather/solar-data/solar-indices

For cycle 24 we calculated FI from Ref. 10 using data on flare activity that are available at

$$
\text { http: //www.wdcb.ru/stp/data/Solar } \text { lare }_{E}{\text { vents } / F l_{X} X I V . p d f}
$$


(see Fig. 2). It was found that the amplitude of FI during cycle 24 decreased by a further factor of 2 compared to cycle 23 .

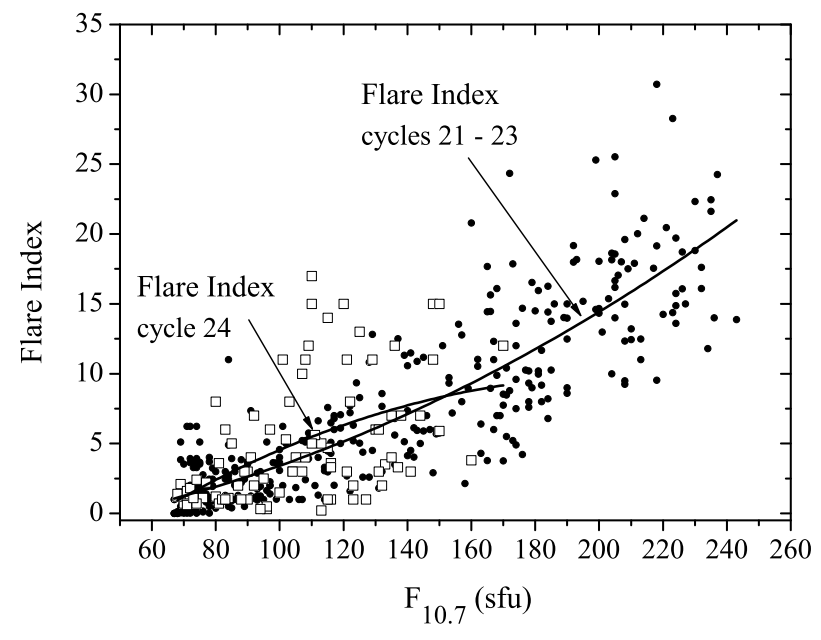

Figure 5: The flare index FI as a function of the radio emission flux at 10.7 $\mathrm{cm}$

Fig. 5 shows the dependence of FI on the radio emission flux at $10.7 \mathrm{~cm}$. The solid points are for cycles 21-23 and the solid squares are for cycle 24 . Quadratic fit curves are shown: the differences in the regression curves for the two dependences are not as marked as in the case of the UV indices, but the spread in the values is greater.

\section{$4 \quad$ Large flares in cycle 24}

Fig. 6 shows the distribution of the MI-X7 (740) flares in cycle 24. The points indicate the months for which the number of flares per month was $\mathrm{N}<6$; the asterisks, the months for which $5<\mathrm{N}<10$; and the large hollow circles, the months for which $\mathrm{N}>20$.

The data for the flares are plotted on a curve showing the monthly-mean numbers of solar spots. It is clear that the largest number of flares took place in the falling branch after the first maximum, and during the second maximum and the falling branch of cycle 24. The largest number of large 
flares occurred in 2014. Before 2011 and since mid-2015, almost no large flares have been observed. As compared to cycles 21-23, where the most powerful flares were observed during the decay phase, in cycle 24 the greatest number of powerful flares (>X2.7) occurred in the rising phase and at the maximum with the exception of the two largest flares of cycle 24, which occurred almost at the minimum between 24 and 25 cycle in September 2017.

\section{North-south (N-S) asymmetry in the local- ization of large flares in cycle 24}

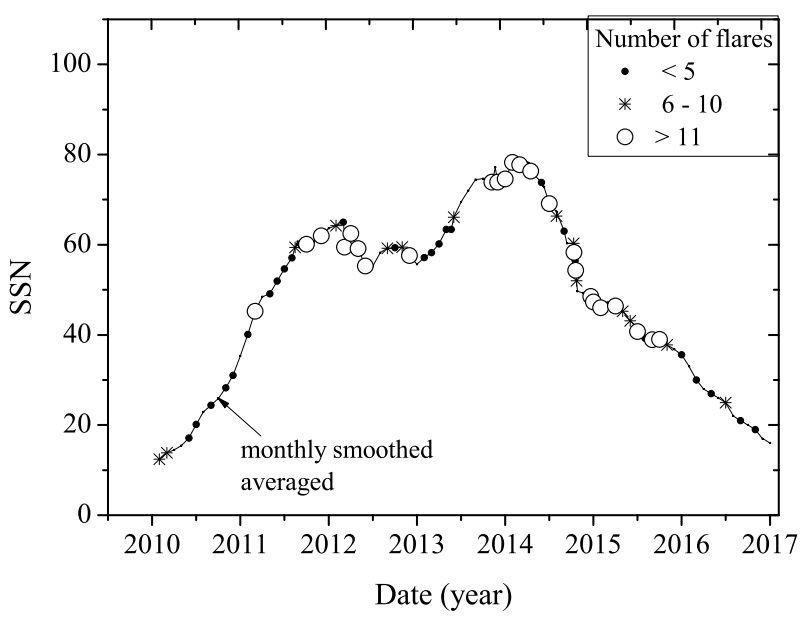

Figure 6: Monthly averaged distribution of large flares during cycle 24

The basic features of the N-S asymmetry have been studied and described in recent years. These show up in all the indices of solar activity, but the reason for this asymmetry is not yet understood. Probably the N-S asymmetry in the indices is a fundamental property of solar activity that is indicative of significant differences in processes taking place in the northern and southern hemispheres. In a study of the N-S asymmetry [11] it was found that many features of the cyclical activity of the sun show up more strongly in the asymmetry of the activity indices than in the values of these indices as such. 
An analysis of solar activity in cycles 21-23 [12] showed that the observed large flares in the soft X-ray range (GOES, 0.1-0.8 nm) in different phases of solar cycles 21-23 had an N-S asymmetry. It was also found that over the entire cycle 21 , a small excess of flare activity was observed in the northern hemisphere, while during cycles 22 and 23, a southern excess predominated. It is important to note that an N-S asymmetry of solar flares shows up both in the number of flares and in the intensities of the flares; this should be taken into account in models of the solar dynamo [12].

We have studied the N-S distribution of flares during cycle 24 .

Table 1 lists data from the analysis of the N-S asymmetry for large flares of X-ray class > M5 (119) in different phases of cycle 24. It is clear that a strong asymmetry in the flare activity was observed during 2011 (15 flares in the northern hemisphere and 4 in the southern) and 2014 (11 in the northern hemisphere versus 28 in the southern). During the rest of cycle 24 , the number of flares in the two hemispheres were roughly equal.

Fig. 7 illustrates the N-S distribution of large flares (monthly average data for X-ray class $>$ M1) in different phases of cycle 24. On the plot of the sliding monthly average numbers of sunspots, different symbols are used to denote the months with distinct N-S asymmetries. In this figure, the solid circles indicate the years when the number of flares in the northern hemisphere is substantially higher than in the southern hemisphere, the asterisks indicate predominant flares in the southern hemisphere, and the hollow circles indicate roughly equal number of flares in the two hemispheres.

It can be seen from Fig. 7 that in the rising phase of cycle 24 the numbers of large flares are initially roughly equal in the northern and southern hemispheres. Then in 2011 and 2012 the number of flares is seen to be larger in the northern hemisphere; this corresponds to an asymmetry in the number of spots.

During the time of the first maximum, a roughly equal number of flares is observed in the two hemispheres. During the second maximum, in the decreasing phase of the cycle from 2013 to 2016, we see that flares predominate in the southern hemisphere; this corresponds to the asymmetry of sunspots during cycles 21-23. In 2016, we again see more flares in the northern hemisphere.

An analysis of the rotation velocities of sunspots and the evolution of the active longitudes [13] shows that the northern and southern hemispheres rotate with different velocities and that the rotation velocities in both hemispheres have increased since 1990. It is assumed that the observed north- 


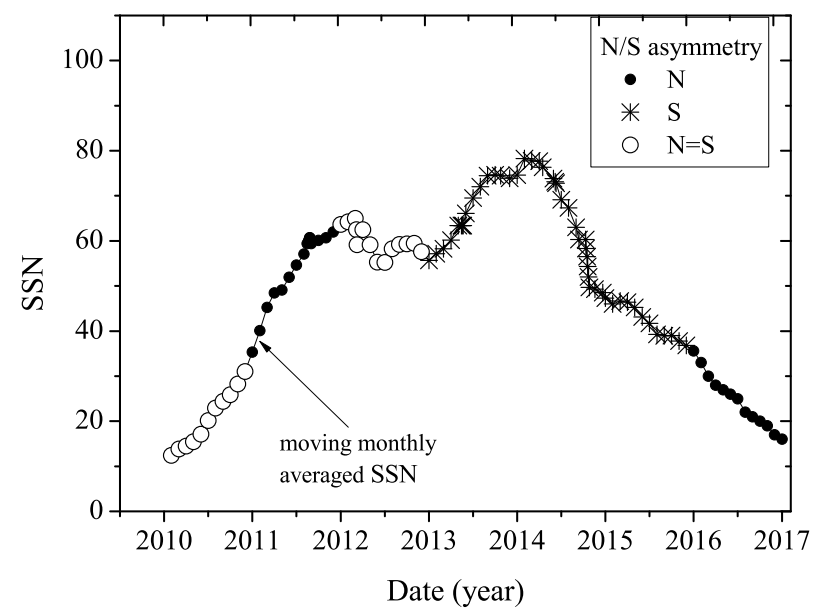

Figure 7: The N-S asymmetry in the number of large $(>\mathrm{M} 1)$ flares during cycle 24

south asymmetries of groups of sunspots and flare activity are consequences of the temporal evolution of the large-scale magnetic field and the solar dynamo.

It is clear that the asymmetry in the flare activity correlates well with the asymmetry in the appearance of groups of sunspots according to data in the archives of the Solar Influences Data Analysis Center (SIDC) of the Royal Observatory of Belgium, which includes the World Data Center for the study of sunspots.

According to these data, for cycles 21-23 and the rising phase of cycle 24, there is an N-S asymmetry in the number of spots that differs slightly from the N-S asymmetry in the flare activity; for all of cycles 21-24 the number of spots in the northern hemisphere predominates in the rising phases of the cycles, while the number of spots in the southern hemisphere predominates in the decreasing phases; see

$$
\text { http : //www.sidc.be/silso/datafiles }
$$

The observations of N-S asymmetry in the various indices are of great significance, since they provide valuable information for modelling the solar dynamo. The appearance of a substantial southern asymmetry at the start 
Table 1: The Asymmetry in Flare Activity During Cycle 24

\begin{tabular}{||c|c|c|c|c||}
\hline \hline Year & $\begin{array}{c}\text { Number flares } \\
>\text { M5 }\end{array}$ & $\begin{array}{c}\text { Number flares } \\
>\text { X1 }\end{array}$ & $\begin{array}{c}\text { Number flares } \\
\text { in N hemisph. }\end{array}$ & $\begin{array}{c}\text { Number flares } \\
\text { in S hemisph. }\end{array}$ \\
\hline 2010 & 3 & 0 & 2 & 1 \\
\hline 2011 & 19 & 8 & 15 & 4 \\
\hline 2012 & 21 & 6 & 12 & 11 \\
\hline 2013 & 21 & 12 & 10 & 28 \\
\hline 2014 & 39 & 16 & 11 & 7 \\
\hline 2015 & 12 & 2 & 5 & - \\
\hline 2016 & 4 & - & 4 & \\
\hline \hline
\end{tabular}

of the 21st century indicates the onset of a period of small activity cycles [14], which we are observing at the present time.

\section{Analysis of satellite observations of the X6.9 flare of August 9, 2011}

UV fluxes have been measured with high accuracy by satellites since 1999 . This has made it possible for us to analyse the onset times in different lines of the largest flare during cycle 24, X 6.9, which took place on August 9, 2011 using observational data from SDO/EVE and GOES-15.

This is important for refining the models of flares. We note the times associated with flare intensification of the fluxes in coronal lines and in lines formed in the chromosphere and transition region. The regions of the coronal flare plasma in which UV and X-ray emission is formed have different heights and temperatures. The gradual increase in the fluxes in these lines during the development of a flare can be used to determine the temperatures within the regions where the flare begins, as well as the in the regions where it propagates.

Fig. 8 shows the evolution of the flare of August 9, 2011, in the coronal lines in the ranges of $9.4,33.5$, and $0.1-0.8 \mathrm{~nm}$, as well as in lines (30.4 and 


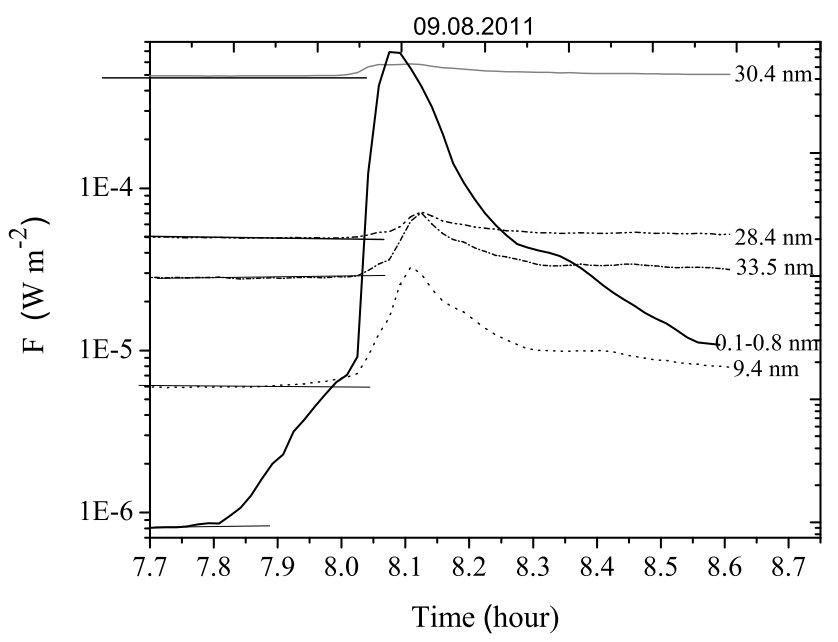

Figure 8: The evolution of the flare of August 9, 2011, in different UV lines and in the X-ray range (the 30.4, 33.5, 28.4, and $9.4 \mathrm{~nm}$ lines and the $0.1-0.8$ nm range)

$28.4 \mathrm{~nm}$ ) of the chromosphere and transition region. The wavelengths are indicated on the right hand side of the figure.

The horizontal smooth lines indicate the level of the background radiation flux; this makes it possible to estimate more accurately the time when the fluxes begin to increase. Note that for shorter wavelengths of a line or spectral range, the ratio of the maximum flare amplitude to the background level is higher. It can be seen that the rise in the fluxes begins gradually (pre-flare enhancement).

In the $0.1-0.8 \mathrm{~nm}$ range and the $9.4 \mathrm{~nm}$ line, the pre-flare begins at $7 \mathrm{~h}$ $48 \mathrm{~m}$ and $7 \mathrm{~h} 54 \mathrm{~m}$, while the pre-flare (which is less distinct) in the other lines begins at $8 \mathrm{~h} 00 \mathrm{~m}$.

Thus, the pre-flare enhancement propagates from the higher levels of the corona into the lower corona and chromosphere. We see the onset of the flare as a sharp, almost vertical increase in the flux for 0.1-0.8 $\mathrm{nm}$ and a steep rise for $9.4 \mathrm{~nm}$ at $8 \mathrm{~h} 02 \mathrm{~m}$, while the onset of the flare for the other lines occurs a few minutes later at $8 \mathrm{~h} 04 \mathrm{~m}-8 \mathrm{~h} 05 \mathrm{~m}$. 


\section{Characteristics of the proton events in cycle 24}

Solar proton events (SPE) and the flares accompanying them during cycle 24 were analysed on the basis of data from GOES 13-15. The most powerful SPE with proton energies $\mathrm{E}>10 \mathrm{MeV}$ and $\mathrm{E}>100 \mathrm{MeV}$ were identified and studied.

SPE are observed in interplanetary space as a result of flares on the sun, as a result of ejection of coronal mass, and sometimes after the disappearance of filaments.

The duration of a proton event depends on the energy of the protons. For energies $10 \mathrm{MeV}$ the duration is a few hours; for higher energies they can last up to several days.

At present there are no unique answers to the question of where, when, and how the protons are accelerated and how they propagate to the observer. The power of proton events is measured in

pfu (particlefluxunits, protons $/ \mathrm{cm}^{2} / \mathrm{s} / \mathrm{sr}$ ).
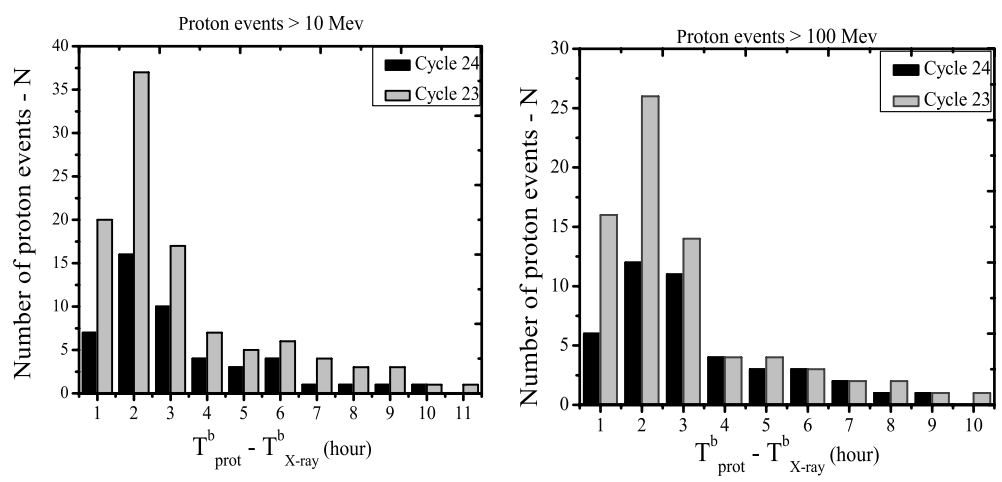

Figure 9: Histogram of the time delays of the start of proton events relative to the onset of the flares that produce the events in the soft X-ray range

Proton events produced by flares and not by other causes (ejection of coronal mass, eruptive prominences) were selected on the basis of the observational data. Proton events in cycles 24 (black) and 23 (grey) were 
Table 2: Flares in Cycle 24 Accompanied by the Largest-scale Proton Events

\begin{tabular}{||c|c|c|c|c||}
\hline \hline Date & X-ray/optic scale & $\begin{array}{c}\text { Position } \\
\text { on the disc }\end{array}$ & $\begin{array}{c}\text { Proton flux } \\
(>10 \mathrm{MeV}), \mathrm{pfu}\end{array}$ & $\begin{array}{c}\text { Proton flux } \\
(>100 \mathrm{MeV}), \mathrm{pfu}\end{array}$ \\
\hline 04.08 .11 & $\mathrm{X} 2.3 / 2 \mathrm{~B}$ & N19W76 & 99 & 2 \\
\hline 23.01 .12 & $\mathrm{M} 8.3 / 2 \mathrm{~B}$ & N28W21 & 4500 & 3 \\
\hline 27.01 .12 & $\mathrm{X} 1.7 / 2 \mathrm{~F}$ & N27W71 & 800 & 11 \\
\hline 07.03 .12 & $\mathrm{X} 5.4 / 2 \mathrm{~B}$ & $\mathrm{~N} 17 \mathrm{E} 27$ & 200 & 70 \\
\hline 07.03 .12 & $\mathrm{X} 1.3 / \mathrm{SF}$ & $\mathrm{N} 23 \mathrm{E} 12$ & 35 & 3 \\
\hline 13.03 .12 & $\mathrm{M} 7.9 / 1 \mathrm{~B}$ & N19W59 & 500 & 2 \\
\hline 17.05 .12 & $\mathrm{M} 5.1 / 1 \mathrm{~F}$ & N11W76 & 250 & 20 \\
\hline 12.07 .12 & $\mathrm{X} 1.4 / 2 \mathrm{~B}$ & N13W15 & 100 & 0.2 \\
\hline 17.07 .12 & $\mathrm{M} 1.7 / 1 \mathrm{~F}$ & S28W90 & 130 & 0.1 \\
\hline 19.07 .12 & $\mathrm{M} 7.7 / \mathrm{SF}$ & S16W90 & 90 & 0.7 \\
\hline 11.04 .13 & $\mathrm{M} 6.5 / 3 \mathrm{~B}$ & N09E12 & 100 & 2 \\
\hline 22.05 .13 & $\mathrm{M} 5.0 / 3 \mathrm{~N}$ & N15W70 & 2000 & 4 \\
\hline 07.01 .14 & $\mathrm{X} 1.2 / 2 \mathrm{~N}$ & S15W11 & 1000 & 4 \\
\hline 10.09 .14 & $\mathrm{X} 1.6 / 2 \mathrm{~B}$ & N12E02 & 130 & 1 \\
\hline 21.06 .15 & $\mathrm{M} 3.8 / 2 \mathrm{~B}$ & S18W57 & 1000 & 0.1 \\
\hline 06.09 .17 & $\mathrm{X} 9.3 / 3 \mathrm{~B}$ & S15W45 & 50 & 0.7 \\
\hline 10.09 .17 & $\mathrm{X} 8.2 / 3 \mathrm{~B}$ & S12W85 & 650 & 70 \\
\hline \hline
\end{tabular}


examined separately. The distributions of the delays between the flare onset in the X-ray range and the start of a proton event are similar in cycles 24 and 23 .

The maxima of the distributions occur for a delay of 2 hours, both for $>10$ $\mathrm{MeV}$ protons and $>100 \mathrm{MeV}$ protons. Note that the most powerful flares are accompanied by proton events with the shortest delays. The largest delays are observed for proton flares lying in the eastern hemisphere because of a longer trajectory for propagation of the protons from the flare region.

Cycle 24 was distinguished by the fact that no proton fluxes with energies $>100 \mathrm{MeV}$ and characterized by a maximum amplitude of more than $100 \mathrm{pfu}$ were observed. During activity cycle 24, the number of flares accompanied by proton fluxes was lower by roughly a factor of 2 .

The characteristics of the energetic solar protons in the first 64 months of cycle 24 (the rising phase and maximum) have been compared with those of the previous cycles (21-23) [15]. It was found that, despite the low solar activity during the rising phase and maximum of cycle 24, the number of SPE with proton energies $\mathrm{E}>10 \mathrm{MeV}$ and $\mathrm{E}>100 \mathrm{Mev}$ in the current cycle differs little from the number of these events in cycles 21-23. During the period from 2010-2016, we identified $62 \mathrm{SPE}$ with proton energies $\mathrm{E}>10 \mathrm{MeV}$ and 24 with $\mathrm{E}>100 \mathrm{MeV}$ (the most powerful of these are listed in Table 2).

Some of the proton events (including the event of March 7, 2012) have a complicated time profile with several maxima and have a high power (see Table 2). It can be seen that a large fraction of these events originated in the northern hemisphere during 2011-2013, while two out of three of the large flares with proton fluxes during 2014-2015 occurred in the southern hemisphere.

It is clear from Table 2 that the largest proton events were associated with flares of class M5 or higher. For the large flares in classes M1-X7 accompanied by fluxes of protons with energies $>10 \mathrm{MeV}$ and $>100 \mathrm{MeV}$, we have analysed the time delay between the onset of a flare in the X-ray range and the onset of the proton event caused by this flare (see Fig. 9). An estimate of the delay in the arrival of the protons in the earth's atmosphere is important for predicting space weather and the state of the upper atmosphere. 


\section{Conclusion}

It follows from this paper that the flare activity during cycle 24 was considerably lower than in cycles 21-23. As compared to cycles 21-23, where the most powerful flares were observed during the decay phase, in cycle 24 the greatest number of powerful flares (>X2.7) occurred in the rising phase and at the maximum with the exception of the two largest flares of cycle 24, which occurred almost at the minimum between 24 and 25 cycles in September 2017.

An analysis of the ultraviolet fluxes in the MgII $280 \mathrm{~nm}$ and La $121.6 \mathrm{~nm}$ lines showed that the regression fits as a function of the overall emission level of the sun differ substantially in cycle 24 and in cycles 21-23.

The evolution of the flare of August 9, 2011, as observed in coronal lines $(9.4,33.5$, and $0.1-0.8 \mathrm{~nm})$ and in lines of the chromosphere and transition region (30.4 and $28.4 \mathrm{~nm}$ ) proceeds in a direction from the upper corona toward the lower corona and chromosphere.

A study of the N-S asymmetry in the flare distribution during cycle 24 showed that a strong N-asymmetry of the flares was observed in 2011 and an S-asymmetry in 2014. In the other years of cycle 24, the numbers of flares in the two hemispheres were roughly the same.

During cycle 24, proton events with $\mathrm{E}>100 \mathrm{MeV}$ are less intense; this confirms the conclusions of Ref. 15. The largest-scale proton events were associated with X-ray flares of class $>$ M5.

Histograms of the time delays of the onset of proton events relative to the onset in the soft X-ray range of the flares causing these events during cycles 24 and 23 show that the maximum of the distribution occurs for a delay of 2 hours for protons with energies $>10 \mathrm{MeV}$, as well as for those with energies $>100 \mathrm{MeV}$.

\section{References}

[1] Kleeorin Y., Safiullin N., Kleeorin N. et al. 2016, MNRAS, 460, 3960.

[2] Gershberg R.E. 2002, Activity of main sequence stars of the sun's type Astroprint, Odessa. 
[3] Shimanovskaya E.V., Bruevich E.A. and Bruevich V.V., 2016, Res. Astron. Astrophys., 16, N9, 148.

[4] Bruevich E.A., Bruevich V.V. and Yakunina G.V. 2014, J. Astrophys. Astron., 35, N1, 1.

[5] Snow M., McClintock W.E., Woods T.N. et al. 2005, Solar Phys., 230, 325.

[6] Bruevich E.A. and Yakunina G.V. 2016, Astrophysics, 59, N3, 369.

[7] Snow M., Weber M.,Machol J., et al. 2014, Space Weather Space Clim., 4, A04.

[8] Bachmann R.T., White O.R. 1994, Solar Phys, 150, 347.

[9] LISIRD - Composite Solar Lyman-alpha, 2016, http://lasp.colorado.edu/lisird/lya.

[10] Atac T., 1987, Astrophys. Space Sci., 135, 201.

[11] Badalyan O.G., 2012, Astron. Lett., 38, 51.

[12] Joshi B., Bhattacharyya R., Pandey K. et al., 2015, Astronomy and Astrophysics, 582, A4.

[13] Zhang L., Mursula K. and Usoskin I., 2011, Astronomy and Astrophysics, 529, A23.

[14] Sykora J., Rybak J., 2010, Solar Phys., 261, 321.

[15] Bazilevskaya G.A., Logachev Yu.I., Bashenyuk E.V. et al., 2015, Izvestiya RAN, seriya fiz., 79, 627. 